Acceleration and Compression of Charged Particle Bunches Using Counter-Propagating Laser Beams

by

G. Shvets, N.J. Fisch, and A. Pukhov

October 2000
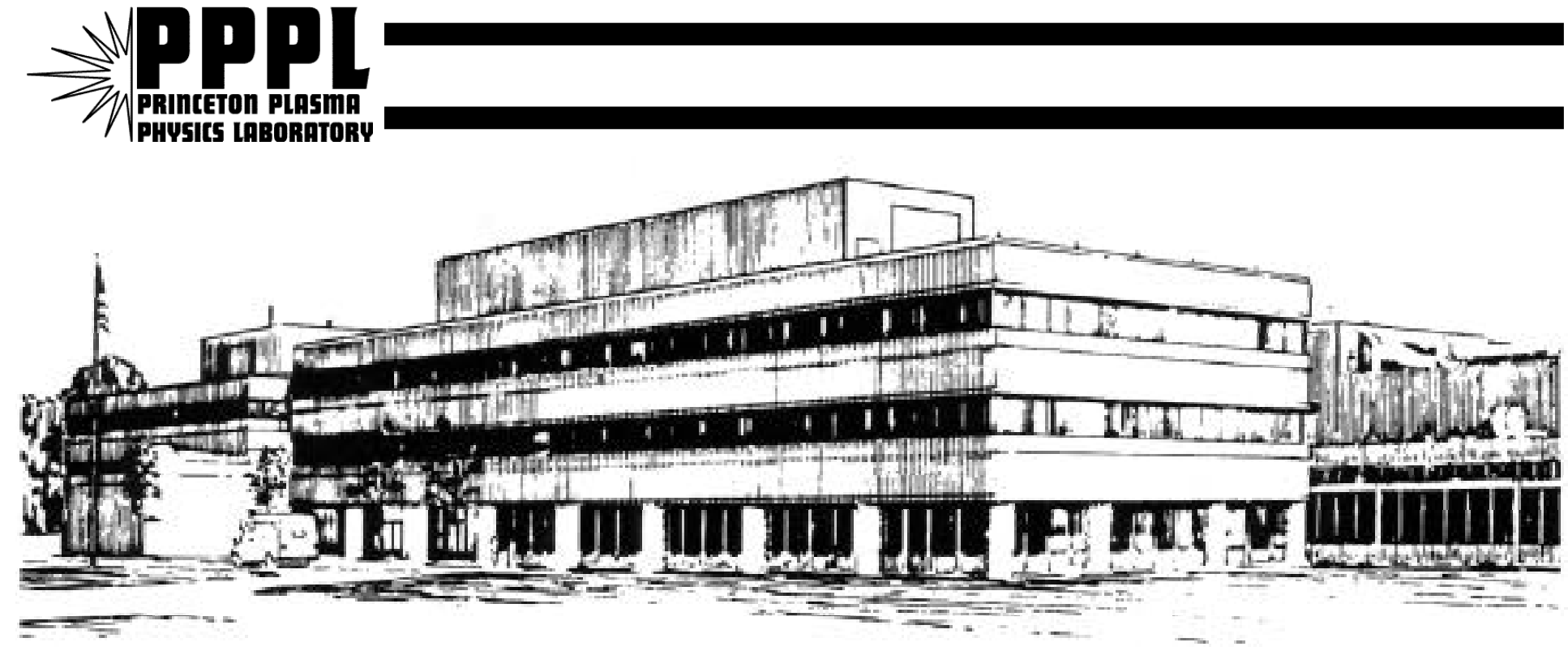

PRINCETON PLASMA PHYSICS LABORATORY PRINCETON UNIVERSITY, PRINCETON, NEW JERSEY 


\section{PPPL Reports Disclaimer}

This report was prepared as an account of work sponsored by an agency of the United States Government. Neither the United States Government nor any agency thereof, nor any of their employees, makes any warranty, express or implied, or assumes any legal liability or responsibility for the accuracy, completeness, or usefulness of any information, apparatus, product, or process disclosed, or represents that its use would not infringe privately owned rights. Reference herein to any specific commercial product, process, or service by trade name, trademark, manufacturer, or otherwise, does not necessarily constitute or imply its endorsement, recommendation, or favoring by the United States Government or any agency thereof. The views and opinions of authors expressed herein do not necessarily state or reflect those of the United States Government or any agency thereof.

\section{Availability}

This report is posted on the U.S. Department of Energy's Princeton Plasma Physics Laboratory Publications and Reports web site in Calendar Year 2000. The home page for PPPL Reports and Publications is: http://www.pppl.gov/pub_report/

DOE and DOE Contractors can obtain copies of this report from:

U.S. Department of Energy

Office of Scientific and Technical Information

DOE Technical Information Services (DTIS)

P.O. Box 62

Oak Ridge, TN 37831

Telephone: (865) 576-8401

Fax: (865) 576-5728

Email: reports@adonis.osti.gov

This report is available to the general public from:

National Technical Information Service

U.S. Department of Commerce

5285 Port Royal Road

Springfield, VA 22161

Telephone: $1-800-553-6847$ or

(703) $605-6000$

Fax: (703) 321-8547

Internet: http://www.ntis.gov/ordering.htm 


\title{
Acceleration and Compression of Charged Particle Bunches Using Counter-Propagating Laser Beams
}

\author{
G. Shvets and N. J. Fisch \\ Princeton Plasma Physics Laboratory, Princeton University, Princeton, NJ 08543 \\ A. Pukhov \\ Max-Planck-Institut für Quantenoptik, D-85748 Garching, Germany
}

\begin{abstract}
The nonlinear interaction between counter-propagating laser beams in a plasma results in the generation of large (enhanced) plasma wakes. The two beams need to be slightly detuned in frequency, and one of them has to be ultra-short (shorter than a plasma period). Thus produced wakes have a phase velocity close to the speed of light and can be used for acceleration and compression of charged bunches. The physical mechanism responsible for the enhanced wake generation is qualitatively described and compared with the conventional laser wakefield mechanism. We also demonstrate that, depending on the sign of the frequency difference between the lasers, the enhanced wake can be used as a "snow-plow" to accelerate and compress either positively or negatively charged bunches. This ability can be utilized in an electron-positron injector.
\end{abstract}

\section{INTRODUCTION}

Plasma was suggested as an attractive medium for particle acceleration [1] because of the high electric field it can sustain. In a plasma-based accelerator [2], particles gain energy from a longitudinal plasma wave whose phase velocity is close to the speed of light. Excitation of such plasma wakes requires a relativistic driver, such as an electron beam in a plasma wakefield accelerator (PWA) [3] or a short laser pulse in a laser wakefield accelerators (LWFA) [1]. The magnitude of the plasma wake in LWFA, measured in units of $E_{\mathrm{wb}}=\mathrm{mc \omega}_{p} / \mathrm{e}$, where $\omega_{p}=\left(4 \pi e^{2} n_{0} / m\right)^{1 / 2}$ is the plasma frequency, is approximately equal to $a_{0}^{2} / 2$, where $a_{0}=e A_{0} / m c^{2}$ is the normalized vector potential of the laser pulse. Here $n_{0},-e$, and $m$ are the plasma electron density, electron charge, and mass, respectively. Since $a_{0}=1$ corresponds to the laser intensity $I_{0}=2.7 \times 10^{18} \mathrm{~W} / \mathrm{cm}^{2} / \lambda_{0}^{2}[\mu \mathrm{m}]$, where $\lambda_{0}=2 \pi c / \omega_{0}$ is the laser wavelength, a relatively high intensity is needed to produce an electric field comparable to $E_{\text {wb }}$.

Recently a novel approach to generating plasma wakes was suggested [4]. It employs two counter-propagating laser pulses: a short timing beam (TB) with frequency $\omega_{0}$, and a long pumping beam (PB) with frequency $\omega_{1}$. To produce a plasma wake of order $e E_{z} / m c \omega_{p} \sim \omega_{p} / \omega_{0}$ the TB has to be shorter than $\omega_{p}^{-1}$ and detuned from the PB by $\left|\Delta \omega=\omega_{0}-\omega_{1}\right| \sim \omega_{p}$. Since this approach requires two colliding laser beams, it is referred to as a colliding beam accelerator (CBA). CBA has two important features: (a) the required intensities of the colliding laser pulses are much smaller than $10^{18} \mathrm{~W} / \mathrm{cm}^{2}$, and (b) the phase of the wake depends on the frequency detuning $\Delta \omega$. Feature (a) can be re-stated as follows: plasma wake due to the low-intensity short laser pulse alone is much smaller than the plasma wake due to the interference of the same short pulse with a long counter-propagating pulse. Therefore, the former wake is referred to as the "regular wake" and the latter wake is referred to as the "enhanced wake".

In this paper we further elucidate the basic differences between the regular and enhanced wakes by exploring the analogy between the plasma wave excitation and the excitation of a pendulum. There are two ways to excite a pendulum: by displacing it and letting go, or by imparting it with momentum through an impulsive kick. It turns out that the excitation of the regular wake is analogous to the former while the excitation of the enhanced wake is analogous to the latter. In addition, we demonstrate how CBA can be used as a compressor/injector of charged particle bunches. Since the phase (or, equivalently, the sign) of the enhanced wake can be controlled by the laser detuning $\Delta \omega$, such an injector can accelerate and compress either electron or positron bunches.

The basic idea of using a laser pulse as a "snowplow" for direct ponderomotive acceleration in a plasma was recently suggested by McKinstrie and Startsev [5]. They found that if the group velocity of the laser pulse $v_{g}<c$, an intensity threshold exists, past which an electron which initially moves with $v_{0}<v_{g}$ can be accelerated to the velocity larger than the laser group velocity. The intensity threshold becomes infinite for a laser pulse moving with the speed of light. For the sub-threshold intensities, the electron is eventually overtaken by the laser pulse without net energy gain. Du and Xu [6] later extended this idea by consistently taking into account the longitudinal electric field of the plasma (although assuming that the laser pulse is longer than the plasma period). They concluded that, under these assumptions, electrons cannot be accelerated by the combined fields of the laser and plasma. Positrons, however, could be accelerated by the leading edge of the electrostatic potential produced in the plasma. What's important, the intensity threshold does not diverge in the limit of $v_{g} \rightarrow c$. Unfortunately, the transverse electric field would sidescatter positrons unless some external focusing is applied. 
Accelerators described in Refs. [5,6], where an injected particle is pushed by the leading edge of either the ponderomotive, or the induced electrostatic potentials, can be referred to as the snow-plow accelerators. They differ from the conventional laser-plasma accelerators in that the final particle energy does not depend on the particle phase with respect to the plasma wake. As long as the intensity threshold is exceeded, the final energy of the particle which is originally ahead of the pulse is determined by the pure kinematics of the collision between a particle and a moving wall. From similar kinematic considerations one concludes that an initially long bunch is compressed in the process of injection.

Although neither of the references $[5,6]$ suggested that a particle injector can be based on their ideas, such practical application is logical if two major drawbacks of the snow-plow acceleration can be overcome: (i) the mechanism only works for positrons, and (ii) transverse electric field defocuses the accelerated particles. In this paper we demonstrate how, in principle, the ideas of snow-plow acceleration and enhanced wake generation can be combined to construct such an injector. To do this, we develop a three-dimensional theory of the CBA. The onedimensional theory was described in Ref. [4]. Other laser injection schemes, not based on the snow-plow acceleration, were recently suggested by Umstadter et. al. [7] and Esarey et. al. [8].

The remainder of the paper is organized as follows. In Section II we present a qualitative one-dimensional analysis and numerical simulation of the colliding beam accelerator. The emphasis is on two properties of the enhanced plasma wake produced in a CBA: (i) large accelerating gradients $(>1 \mathrm{GeV} / \mathrm{m})$ can be obtained using laser pulses of sub-relativistic $\left(\ll 10^{18} \mathrm{~W} / \mathrm{cm}^{2}\right)$ intensities, and (ii) the phase of the enhanced wake can be changed by $\Delta \phi=\pi$ by simply changing the sign of the frequency detuning between the timing and pumping beams. In Section III we review the basics of the snow-plow acceleration in one dimension. The three dimensional theory of the colliding beam accelerator in an almost-homogeneous plasma is derived in Section IV. Section V concludes.

\section{ONE-DIMENSIONAL THEORY OF COLLIDING BEAM ACCELERATOR}

To illustrate the concept of the colliding beam accelerator, the following physical problem was simulated using a one-dimensional particle-in-cell (PIC) code VLPL [9]. An ultra-short circularly polarized Gaussian laser pulse with duration $\tau_{L}=1.5 \omega_{p}^{-1}$ and normalized vector potential $a_{0}=0.12$, propagating in the positive $z$ direction, collides in a plasma with a long counter-propagating pulse with $a_{1}=0.05$. Plasma density was chosen such that $\omega_{p} / \omega_{0}=0.05$. The snapshot of the pulse intensity normalized to $2.7 \times 10^{18} \mathrm{~W} / \mathrm{cm}^{2}$ is shown in Fig. 1(i).
Two cases, corresponding to the different frequencies of the PB, $\omega_{1}=1.1 \omega_{0}$ and $\omega_{1}=0.9 \omega_{0}$, were simulated. The resulting plasma wakes are shown in Fig. 1(iii) and (iv), respectively. For comparison, we also plot the wake produced by a single TB in absence of the counterpropagating pulse in Fig. 1(ii).

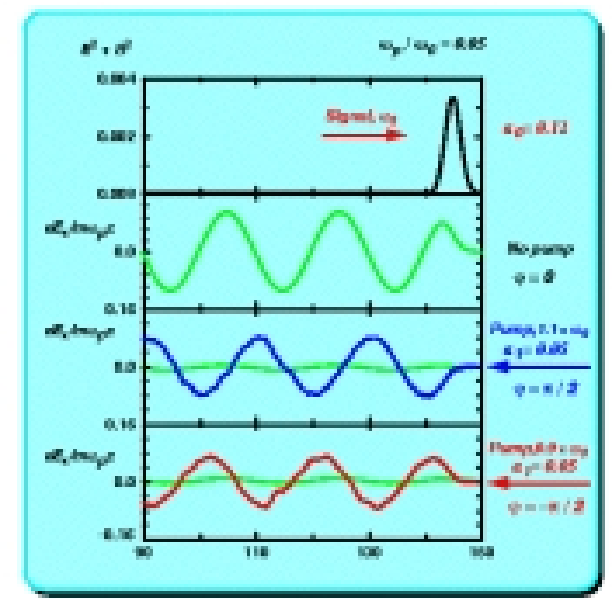

FIG. 1. Top to bottom: (i) single short laser pulse with $a_{0}=0.12$ and frequency $\omega_{0}$ propagates from left to right; (ii) short pulse generates a weak plasma wake $E_{x}$; (iii) in the presence of counter-propagating pump with $a_{1}=0.05$ and frequency $\omega_{1}=1.1 \omega_{0}$ the wake is enhanced, and its phase is shifted by $\pi / 2$ with respect to the "regular" wake of (ii), which is also shown for comparison; (iv) Same as (iii), only a down-shifted pump with $\omega_{1}=0.9 \omega_{0}$ is used, and the phase shift is $-\pi / 2$.

Since the intensity of the short pulse is chosen nonrelativistic, the magnitude of the plasma wake left behind the pulse is much smaller than the limiting (wavebreaking) field according to $E / E_{\mathrm{wb}} \sim a_{0}^{2} / 2$. The situation changes dramatically when a counter-propagating beam is added. As Figs. 1(iii) and (iv) indicate, the addition of the pumping beam increases the electric field of the plasma wake by an order of magnitude. To further illustrate this point, we plotted the regular wake [same as shown in Fig. 1(i)] in Figs. 1(iii-iv) for comparison. Note that the vertical scales of the Figs. 1(iii-iv) and Fig. 1(ii) differ by a factor 20 . Plasma wakes produced as a result of the collision between the counter-propagating beams is referred to as the enhanced wake because it can be much larger than the regular wake.

The origin of the enhanced wake can be qualitatively understood by comparing its phase with that of the regular wake. As the comparison of Figs. (ii) and (iii) indicates, the phase of the enhanced wake differs by $\Delta \phi=\pi / 2$ from that of the regular wake. Recall that when a laser pulse of the duration $\tau_{L}<\omega_{p}^{-1}$ interacts with a plasma electron, an electron is displaced by $\xi_{0}=c \int d \zeta\left|a_{0}\right|^{2} / 2[10]$, where $\zeta=t-z / v_{g}$. The momentum gained by the electron from the laser pulse is small 
since the plasma response can be neglected for short pulse durations. Here, and elsewhere we assume nonrelativistic laser intensities $a_{0,1} \ll 1$ for both the short and long pulses. Plasma is assumed tenuous, $\omega_{p} \ll \omega_{0}$, so that $\zeta \approx t-z / c$.

The above reasoning applies not only to a single plasma electron but also to any small fluid element of the plasma. Fluid description is appropriate here because the electron displacement $\left|k_{p} \xi\right| \ll 1$ for a short laser pulse of nonrelativistic intensity. Therefore, electron trajectories do not cross, and the Lagrangian displacement of the fluid element at $z=z_{0}$ satisfies the harmonic oscillator equation $\ddot{\xi}+\omega_{p}^{2} \xi=0$. The initial condition $\xi\left(z_{0}, t=z_{0} / c\right)=\xi_{0}$ and $\dot{\xi}\left(z_{0}, t=z_{0} / c\right)=0$ is given along the world line of the short laser pulse which sets up the initial displacement $\xi_{0}$. The solution of the harmonic oscillator equation which satisfies this initial condition is $\xi=\xi_{0} \cos \omega_{p}\left(t-z_{0} / c\right)$. Electric field of the resulting plasma wake is found from $E_{z}=4 \pi e n_{0} \xi$ :

$$
\frac{e E_{z}}{m c \omega_{p}}=\frac{\omega_{p} \xi_{0}}{c} \cos \omega_{p} \zeta=\left(\frac{\sqrt{\pi}\left|a_{0}\right|^{2}}{2}\right) \cos \omega_{p} \zeta,
$$

where we assumed that $a_{0}^{2} \propto \exp \left[-\zeta^{2} / \tau_{L}^{2}\right]$.

Enhanced wake is shifted in phase by $\pi / 2$ with respect to the regular wake because the initial conditions for every fluid element along the path of the short pulse are set up differently. Assume, for example, that every plasma fluid element is imparted with an initial velocity $\xi_{0}$ instead of being initially displaced. With such an initial condition, the solution of the harmonic oscillator equation, $\xi=\left(\dot{\xi}_{0} / \omega_{p}\right) \sin \omega_{p}\left(t-z_{0} / c\right)$, exhibits the observed from the simulations phase shift. The initial velocity is imparted to the plasma via the backscattering of the long pulse into the short one (or vice versa, depending on the frequency detuning $\Delta \omega$ ), as explained below.

When two counter-propagating laser pulse interfere, an almost-standing intensity wave is produced. Its spatial and temporal periods are $2 \pi /\left(k_{0}+k_{1}\right) \approx \pi / k_{0}$ and $2 \pi / \Delta \omega$, respectively. This intensity grating is slowly moving with the speed $v_{\mathrm{ph}}=\Delta \omega /\left(2 k_{0}\right) \ll c$, and in the limit of the monochromatic beams becomes a standing wave. Plasma electrons are affected by the ponderomotive force $\vec{F}=-\nabla \Phi_{p}$ which is proportional to the gradient of the ponderomotive potential $\Phi_{p}=m c^{2}\left|a^{2}\right| / 2$, created by the interference of the two lasers. This ponderomotive force, directed along the $z$-axis, is given by $F_{p}=2 k_{0} a_{0} a_{1} \cos \left(2 k_{0} z-\Delta \omega t\right)$.

It is convenient to introduce an electron ponderomotive phase $\psi_{j}=2 k_{0} z_{j}-\Delta \omega t$ of the $j$ 'th particle inside a given ponderomotive period. Using this definition and the expression for the ponderomotive force, a useful quantity $\omega_{B}^{2}=4 \omega_{0}^{2} a_{0} a_{1}$ can be constructed which has the dimension of the frequency squared, and characterizes the strength of the ponderomotive force. If $\omega_{B}^{2}>\omega_{p}^{2}$, then the Coulomb interactions between the plasma electrons can be neglected in comparison with the ponderomotive force. Equation of motion for the $j$ 's electron in this case is particularly simple:

$$
\ddot{\psi}_{j}+\omega_{B}^{2}(\zeta) \sin \psi_{j}=0
$$

where, as before, a dot denotes a derivative with respect to time $t$ or the co-moving coordinate $\zeta=t-z / c$. The functional dependence of $\omega_{B}$ on $\zeta$ is determined by the longitudinal profile of the short pulse; the bounce frequency vanishes before and after the timing beam.

Plasma electrons, initially stationary in the laboratory frame, enter the time-dependent ponderomotive bucket with the initial "speed" $\dot{\psi}=-\Delta \omega$. If this speed is smaller than the bucket height $\dot{\psi}_{\max }=2 \omega_{B}$, some electrons (with the appropriate initial ponderomotive phase) become trapped and execute a synchrotron oscillation in the bucket. It turns out that, by appropriately choosing the pulse duration and frequency detuning, a substantial average momentum $P_{z}$ can be imparted to plasma electrons. Clearly, depending on their initial phase $\psi_{j}$, some electrons will gain positive or negative momentum. The emphasis, however, is on the average momentum transferred to a fluid element of size $\lambda_{0} / 2$. The sign and magnitude of $P_{z}$ varies on a scale of the short pulse duration which is assumed much larger than $\lambda_{0} / 2$. This constitutes the major difference between the generation mechanisms of the regular and enhanced wakes. In the case of a regular wake the fluid description on all spatial scales is completely adequate. In the case of an enhanced wake fluid description is sufficient on a long spatial scale (of order $c \tau_{L}$ or $\left.c / \omega_{p}\right)$. Fully kinetic treatment may be necessary on a short scale of the ponderomotive period.

To model the electron dynamics on a scale of one ponderomotive period, we assume that the TB has a Gaussian temporal profile, $\omega_{B}^{2}(\zeta) \equiv \omega_{B}^{2} \exp \left(-\zeta^{2} / 2 \tau_{L}^{2}\right)$, and solve the nonlinear pendulum equation (2) for an ensemble of test electrons. Before the arrival of the TB (at $\zeta=-\infty)$ the electrons are uniformly distributed in phase $0<\psi_{j}<2 \pi$ and have identical $\dot{\psi}_{j}=-\Delta \omega$. The average momentum $P_{z}$, gained by the electrons after the interaction, is calculated as $P_{z}=\left(m / 2 k_{0}\right) \sum_{j} \Delta \dot{\psi}_{j}$, where $\Delta \dot{\psi}_{j}=\dot{\psi}_{j}(\zeta=+\infty)-\dot{\psi}_{j}(\zeta=-\infty)$. In the color plot of Fig. 2, $P_{z}$ is shown as the function of the normalized pulse duration $\omega_{B} \tau_{L}$ and frequency detuning $\Delta \omega / \omega_{B}$. From Fig. 2, the average momentum, gained by the electrons, has the same sign as the frequency detuning.

The largest average momentum gain $P_{z} \approx m c \Delta \omega / \omega_{0}$ is realized for $\Delta \omega \approx \omega_{B}$ and $\tau_{L} \approx 2 / \omega_{B}$. For these parameters, most of the electrons execute half of a bounce in the ponderomotive bucket. Other bright color streaks in Fig. 2 correspond to the electrons executing $3 / 2,5 / 2$, etc. bounces. For those higher-order resonances $P_{z}$ is maximized for longer pulse durations $\tau_{L}$. If the pulse duration is longer than $\omega_{p}^{-1}$, the neglected space-charge 
terms are likely to wash out these higher-order resonances and to reduce $P_{z}$. The nonzero momentum transfer to the plasma electrons is due entirely to the short duration of the TB. While it was long recognized [11] that the interaction between laser beams in the plasma results in the momentum transfer to plasma electrons and ions, most calculations assumed long laser beams, in which case most of the momentum was transferred to the ions.

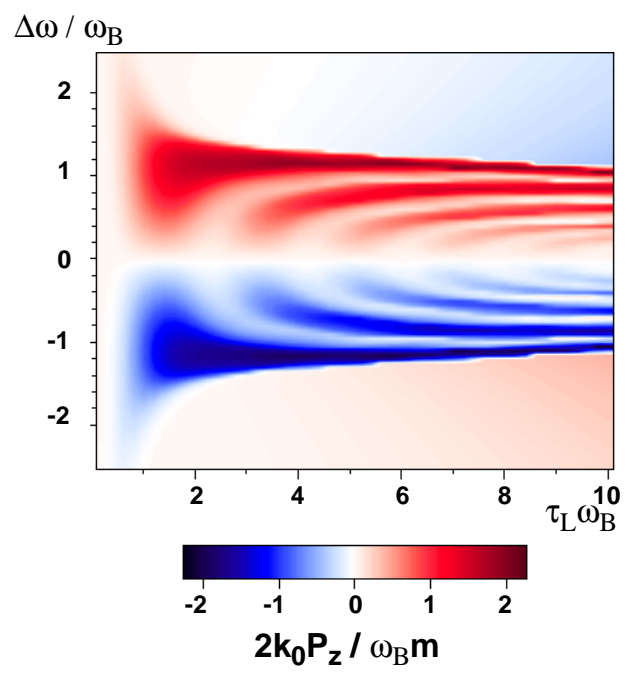

FIG. 2. Average momentum $P_{z}$ gained by plasma electrons in the ponderomotive bucket as a function of the normalized TB duration $\omega_{B} \tau_{L}$ and the frequency detuning $\Delta \omega / \omega_{B}$.

The kinetic modeling presented above provides the averaged value of the transferred momentum as an input for a long-scale fluid calculation. Namely, with an initial condition for the fluid element velocity $\dot{\xi}_{0}=P_{z} / \mathrm{m}$, we calculate the electric field of the enhanced wake as

$$
\frac{e E_{z}}{m c \omega_{p}}=\frac{\left\langle P_{z}\right\rangle}{m c} \sin \omega_{p} \zeta \approx\left(\frac{\Delta \omega}{\omega_{0}}\right) \sin \omega_{p} \zeta
$$

In deriving Eq. (3) we assumed that the initial displacement of the fluid element $\xi_{0}=0$. This is because, as one observes from Eq. (2), plasma electrons $k$ and $j$ are "clamped" at the opposite sides of the ponderomotive period, $\psi_{k}=0$ and $\psi_{j}=2 \pi$. Comparison between Eqs. $(1,3)$ indicates that the phase shift between the regular and the enhanced wakes is $\pi / 2$, just as observed from Figs. 1(ii) and (iii). Moreover, changing the sign of the frequency detuning $\Delta \omega$ shifts the phase of the enhanced wake by $\pi$, in agreement with the simulations results from Figs 1(iii) and (iv).

To conclude this section, we present an alternative derivation of the enhanced wake in one dimension which will be extended to three dimensions in Section IV. The electric field of the enhanced wake can be interpreted as a displacement current. As the photons are exchanged between the counter-propagating beams, electrons, on average, acquire the recoil momentum $P_{z}$ and produce a nonlinear current $J_{\mathrm{NL}}=-e n_{0} P_{z} / m$. In 1-D any current flowing through the plasma must be balanced by the displacement current [11]. Therefore, an electric field $E_{z}$ is produced, satisfying Faraday's law $\partial E_{z} / \partial t=-4 \pi J_{z}$, where $J_{z}$ is the combined current in the plasma. Two flows contribute to $J_{z}$ : the linear plasma flow in the field of the enhanced wake $J_{f}=-e n_{0} v_{f}$, and the nonlinear (space-averaged) flow $J_{\mathrm{NL}}$. Taking the time derivative of Faraday's law (with $\nabla \times \vec{B}=0$ ), we obtain

$$
\left(\frac{\partial^{2}}{\partial \zeta^{2}}+\omega_{p}^{2}\right) E_{z}=-4 \pi \frac{\partial J_{\mathrm{NL}}}{\partial \zeta}
$$

The earlier Eq. (3) for the enhanced wake can be recovered from Eq. (4) by assuming that $J_{\mathrm{NL}}(\zeta)=$ $-e n_{0} P_{z} / m H(\zeta)$. This assumption is justified when the TB duration is shorter than $\omega_{p}^{-1}$.

\section{SNOW-PLOW ACCELERATION: ONE-DIMENSIONAL CALCULATION}

In this Section we explain how a combination of the ponderomotive force of a short laser pulse and plasma wake can be used to accelerate charged particles to the velocity exceeding the group velocity of a laser pulse in the plasma. The original idea of using ponderomotive force of the leading edge of a laser pulse to accelerate a charged particle (electron or positron) was suggested by McKinstrie and Startsev [5]. They found that if the particle is initially ahead (to the right) of the laser pulse moving with the group velocity $v_{g}=\beta_{g} c$, and its initial velocity $v_{0}=\beta_{0} c<v_{g}$, then if the intensity of the laser pulse is sufficiently high, the particle can bounce off the laser pulse, gaining energy as the result. This calculation can be extended to the case of the laser pulse moving through the plasma.

In the reference frame moving with $v_{g}$ the initial energy-momentum of the particle are $\gamma^{\prime}=\gamma_{g} \gamma_{0}\left(1-\beta_{g} \beta_{0}\right)$ and $p^{\prime}=\gamma_{g} \gamma_{0}\left(\beta_{0}-\beta_{g}\right)$. Primed variables are calculated in the reference frame moving with the pulse. In the pulse frame the particle velocity is negative if in the laboratory frame $\beta_{0}<\beta_{g}$. However, if the pulse is sufficiently intense, particle can reflect off the pulse, reversing the sign of its momentum in the pulse frame. Transforming back to the laboratory frame, we find that, after reflection, the final particle energy is

$$
\gamma_{f}=\gamma_{0} \gamma_{g}^{2}\left(1+\beta_{g}^{2}-2 \beta_{g} \beta_{0}\right)
$$

The final energy does not depend on the exact details of the force experienced by the particle: in one dimension energy and momentum can be expressed as functions of the interaction potential which vanishes before and after the interaction. Therefore, expression (5) is simply a consequence of the conservation of energy and momentum. 
For a laser pulse in vacuum McKinstrie and Startsev found that a threshold laser intensity needed for reflecting an electron (or positron) is given by $a_{0}^{2}=$ $\gamma_{g}^{2} \gamma_{0}^{2}\left(1-\beta_{g} \beta_{0}\right)^{2}-1$. This estimate also holds for a tenuous plasma provided that the pulse is shorter than $\omega_{p}^{-1}$. Note that for a pulse moving with a speed of light the threshold intensity diverges.

In the presence of a dense plasma the threshold for electrons is further increased because the ponderomotive pressure of the laser pulse on the ambient electron plasma leads to charge separation. The resulting electrostatic potential effectively decreases the ponderomotive potential of the laser pulse experienced by the electrons injected for acceleration. The situation is, however, reversed for positrons, as was pointed out by Du and Xu [6]. Moreover, since in the case of positrons injected into electron plasma acceleration is done directly by the electric field, not by the ponderomotive force, the laser intensity threshold for snowplowing positrons remains finite. To see why this is the case, consider the one-dimensional equations of motion for a particle with charge $q= \pm e$ and mass $m$.

By conservation of the transverse canonical momentum, $\vec{v}_{\perp}=-(q / e) \vec{a} / \gamma$, where $\gamma=\left(1+a^{2}+u_{z}^{2}\right)^{1 / 2}$, and $u_{z}=\gamma \beta_{z}$. The corresponding equations for $u_{z}$ and $\gamma$ become

$$
\begin{aligned}
& \frac{d u_{z}}{d t}=-c \frac{\partial \tilde{\Phi}}{\partial z}-\frac{c}{2 \gamma} \frac{\partial|a|^{2}}{\partial z} \\
& \frac{d \gamma}{d t}=-\frac{c u_{z}}{\gamma} \frac{\partial \tilde{\Phi}}{\partial z}+\frac{1}{2 \gamma} \frac{\partial|a|^{2}}{\partial t}
\end{aligned}
$$

where the electric field is proportional to the derivative of $\tilde{\Phi}: E_{z}=-\left(m c^{2} / q\right) \partial \tilde{\Phi} / \partial z$. In a $1-\mathrm{D}$ plasma wake all fields are functions of $\zeta$ alone, so the normalized potential $\tilde{\Phi}$ can always be introduced as an integral of $E_{z}$ over $\zeta$.

The first terms in the RHS of Eqs. $(6,7)$, which describe the electric field of the wake, dominate over the second (ponderomotive) terms for large $\gamma$. Therefore, in the remainder of the paper we neglect the direct ponderomotive force. The opposite limit of $\gamma_{g} \tilde{\Phi}<\left|a^{2}\right|$ was taken in Ref. [5], where the vacuum threshold intensity was obtained. The complete system of equations $(6,7)$, along with the Poisson's equation for $\tilde{\Phi}$, was earlier considered by Esarey and Sprangle [12].

From the invariant of the equations of motion $\gamma+\tilde{\Phi}-$ $\beta_{g} u_{z}=\gamma_{0}\left(1-\beta_{0} \beta_{g}\right)$ and $\gamma^{2} \approx 1+u_{z}^{2}$ (we dropped the $\left|a^{2}\right|$ term) particle momentum can be expressed as a function of $\tilde{\Phi}$ :

$$
\begin{aligned}
& u_{z}=\gamma_{g}\left[\beta_{g} \gamma_{g}\left(C_{0}-\tilde{\Phi}\right) \pm \sqrt{\gamma_{g}^{2}\left(C_{0}-\tilde{\Phi}\right)^{2}-1}\right] \\
& \gamma=\gamma_{g}\left[\gamma_{g}\left(C_{0}-\tilde{\Phi}\right) \pm \beta_{g} \sqrt{\gamma_{g}^{2}\left(C_{0}-\tilde{\Phi}\right)^{2}-1}\right]
\end{aligned}
$$

where $C_{0}=\gamma_{0}\left(1-\beta_{g} \beta_{0}\right)$. The plus (minus) sign corresponds to a particle which is moving faster (slower) than the laser pulse. The switching from minus to plus signs takes place when the expression under the square root vanishes. This happens at $\zeta_{r}$ which corresponds to $\tilde{\Phi}\left(\zeta_{r}\right)=C_{0}-\gamma_{g}^{-1}$. The accelerating potential has to be larger than this threshold value to ensure the snow-plow acceleration. It can be shown that $C_{0}-\gamma_{g}^{-1}>0$, so that a positive (negative) potential is required for accelerating positrons (electrons).

The most interesting case is $\gamma_{0} \ll \gamma_{g}$. The final energy of the accelerated particle is then approximately given by $\gamma_{f}=\gamma_{g}^{2} / \gamma_{0}$. The threshold condition for snow-plow acceleration also simplifies to $\tilde{\Phi}>\left(2 \gamma_{0}\right)^{-1}$. Therefore, the initially fast particles with $1 \ll \gamma_{0} \ll \gamma_{g}$ are easier to trap than the stationary ones. The relative energy boost $\gamma_{f} / \gamma_{0}$ is, however, decreased in the same proportion.

The relative energy boost is related to the bunch compression ratio. By conservation of the longitudinal phase space, $l_{f} \delta \gamma_{f}=l_{0} \delta \gamma_{0}$, where $l_{f}$ and $l_{0}$ are the final and initial bunch lengths, and $\delta \gamma_{f}$ and $\delta \gamma_{0}$ are the final and initial energy spreads, respectively. Since the final energy scales as $\gamma_{0}^{-1}$, the final and initial energy spreads are related through $\left|\delta \gamma_{f} / \delta \gamma_{0}\right|=\gamma_{f} / \gamma_{0}$ Therefore, both the energy can be boosted and the bunch length decreased by a large factor $\gamma_{g}^{2} / \gamma_{0}^{2}$ if $\gamma_{0} \ll \gamma_{g}$.

One arrives at the same result by requiring that the beam density remains unchanged in the reference frame of the laser pulse, where a particle bunch is scattered by a stationary potential. Using Lorenz transformation, the particle density $n^{\prime}$ in the pulse frame can be expressed in terms of the initial and final densities $n_{0}$ and $n_{f}$, respectively:

$$
\gamma_{g} n_{0}\left(1-\beta_{0} \beta_{g}\right)=n^{\prime}=\gamma_{g} n_{f}\left(1-\beta_{f} \beta_{g}\right) .
$$

Therefore, $n_{f} / n_{0}=\left(1-\beta_{0} \beta_{g}\right) /\left(1-\beta_{f} \beta_{g}\right)$. This ratio is equal to $\gamma_{f} / \gamma_{0}$ since the particle energy in the primed frame remains the same before and after the reflection: $\gamma_{g}\left(\gamma_{0}-\beta_{g} u_{0}\right)=\gamma_{g}\left(\gamma_{f}-\beta_{g} u_{f}\right)$. The bunch compression ratio $l_{f} / l_{0}$ is then given by $l_{f} / l_{0}=n_{0} / n_{f}=\gamma_{0} / \gamma_{f}$.

One possible application of the snow-plow acceleration is an injector capable of accelerating and compressing initially low-energy long particle bunches. Since the ability to inject both electrons and positrons is crucial, such an injector should be able to produce positive as well as negative potential wells. Colliding beam accelerator can accomplish just that by adjusting the frequency detuning $\Delta \omega$ between lasers. The examples of the electron and positron injectors are shown in Figs. 1(iii) and (iv), respectively. Note an important difference in the dynamics of the cold ambient plasma electrons and the initially relativistic electrons injected into the plasma just ahead of the laser pulse. While the slow plasma electrons experience the beatwave of the counter-propagating laser pulses, the fast electrons are barely affected by the beatwave ponderomotive force because the phase velocity of the beatwave is much smaller than the speed of light. 
Let's calculate the maximum normalized potential $\tilde{\Phi}$ produced in a CBA using Fig. 1(iii) as a blueprint for an electron injector. Assuming $\Delta \omega \approx-\omega_{p}$ and using Eq. (3), obtain, for injected electrons, $\tilde{\Phi} \approx \omega_{p} / \omega_{0}(1-$ $\left.\cos \omega_{p} \zeta\right)$. Therefore, the condition for the snow-plow acceleration becomes $2 \omega_{p} / \omega_{0}>\left(2 \gamma_{0}\right)^{-1}$, or $\gamma_{0}>\omega_{0} / 4 \omega_{p}$. The presence of the plasma naturally reduces the group velocity of the laser pulse: $\gamma_{g}=\omega_{0} / \omega_{p}$. Therefore, if the initial energy of the electron bunch is inside the interval $\omega_{0} / 4 \omega_{p}<\gamma_{0}<\omega_{0} / \omega_{p}$, CBA can be used for snow-plow acceleration/bunch compression.

To calculate the interaction distance required to accomplish the snow-plow acceleration, approximate the normalized potential by a linear function, assuming for simplicity that $C_{0}-\tilde{\Phi} \equiv X=C_{0}\left(1-\zeta / \tau_{g}\right)+\gamma_{g}^{-2}$, where $\tau_{g}=\pi / \omega_{p}$ is a typical gradient scale, approximately set equal to $\omega_{p}^{-1}$. The reflection point $\zeta_{r}=\tau_{g}$, and the starting (and final) point is $\zeta \approx 0$. Expanding Eqs. $(8,9)$ in the limit of $\beta_{g} \approx 1$ and using $\dot{\zeta}=1-u_{z} /\left(\beta_{g} \gamma\right)$, we obtain the following equation of particle motion relative to the moving potential:

$$
\frac{d \zeta}{d t}=\frac{\sqrt{X^{2}-\gamma_{g}^{-2}}\left(X+\sqrt{X^{2}-\gamma_{g}^{-2}}\right)}{1+X^{2}},
$$

which is solved with initial condition $\zeta(t=0)=0$ (or $\left.X=C_{0}\right)$. The time it takes a particle to reach $\zeta=\tau_{g}$ and return back to $\zeta=0$ is given by

$t_{\mathrm{ac}}=\left(\frac{2 \tau_{g} \gamma_{g}^{2}}{C_{0}}\right) \int_{\gamma_{g}^{-1}}^{C_{0}} \frac{d X \quad X}{\sqrt{X^{2}-\gamma_{g}^{-2}}}=2 \tau_{g} \gamma_{g}^{2} \sqrt{1-C_{0}^{-2} \gamma_{g}^{-2}}$

In the limit of $\gamma_{g} C_{0} \gg 1$ we find that $t_{\mathrm{ac}} \approx 2 \tau_{g} \gamma_{g}^{2}$. To gain more physical insight into this result, note that average acceleration gradient is equal to the $\langle W\rangle=$ $m c^{2} \gamma_{f} /\left(c t_{\mathrm{ac}}\right)=m c^{2} C_{0} /\left(c \tau_{g}\right)$, which is precisely the magnitude of the electric field.

Consider the following numerical example: plasma with density $n_{0}=2.5 \cdot 10^{18} \mathrm{~cm}^{-3}$ (corresponding to $\omega_{0} / \omega_{p}=20$ ) is used as a medium for compressing a 3.2 $\mathrm{MeV}$ electron bunch of $1 \mathrm{ps}$ duration. Since $\gamma_{g}^{2} / \gamma_{0}^{2}=10$ for this example, the output of such an injector is a 100 $\mathrm{fs}, 32 \mathrm{MeV}$ electron bunch. The required plasma length is estimated $c t_{\mathrm{ac}}=2.6 \mathrm{~mm}$ by assuming that $\tau_{g}=\omega_{p}^{-1}$.

One dimensional treatment of a snow-plow injector is sufficient only to determine its accelerating properties. Full three-dimensional description of the transverse electric and magnetic fields of the injector is needed to study the transverse particle dynamics. It is essential that the accelerated particles are focused to (or, at least, not defocused from) the laser axis. As McKinstrie and Startsev point out in Ref. [5], one of the limitations of the direct laser acceleration by the leading edge of the pulse is that the particles are scattered off-axis, and a very wide laser beam is needed to prevent particles from scattering out of the beam. The same drawback is found in the positron injector scheme of Du and Xu [6], where a long $\left(\gg \omega_{p}^{-1}\right)$ laser pulse is used to adiabatically produce an electrostatic potential $\tilde{\Phi}$. Since the shape of this potential follows the shape of the laser intensity, it is peaked on axis, causing positrons to scatter out of the beam.

Although in this section we used the potential description of the longitudinal (accelerating) electric field, this was only done for notational convenience: an integral $\tilde{\Phi}=(q / m c) \int d \zeta E_{z}$ can always be introduced whether the electric field is curl-free (electrostatic) or not. In one dimension the $E$-field is always curl-free, so there is no need to introduce the distinction between the actual scalar potential and the quantity $\tilde{\Phi}$ defined above - both are the same. If the electric field is electrostatic in $3-$ $D$, and the accessible region for positrons (electrons) is $\tilde{\Phi}>0(\tilde{\Phi}<0)$, then particles are going to be defocused from the axis if $|\tilde{\Phi}|$ peaks on-axis. In three dimensions the enhanced wakefield is not curl-free. The full 3-D theory of CBA is developed in the next Section.

\section{THREE-DIMENSIONAL THEORY OF CBA}

At the end of Section II we presented an alternative derivation of the equation for the electric field in a CBA. This derivation was based on splitting the total current $\vec{J}$ flowing through the plasma into two components: the linear plasma flow in the field of the enhanced wake $\vec{J}_{f}=-e n_{0} \vec{v}_{f}$ and nonlinearly-generated recoil current $\vec{J}_{\mathrm{NL}}=-\hat{z} e n_{0} P_{z} / m$ which is produced via the photon exchange between the counter-propagating laser beams. Plasma flow velocity $\vec{v}_{f}$ satisfies the linearized equation of motion $\partial \vec{v}_{f} / \partial t=-e \vec{E} / m$, where $\vec{E}$ is the enhanced wake field. In Section II we assumed that $\vec{B}=0$, as appropriate for a one-dimensional calculation. In this section we keep the magnetic field which is crucial for determining the focusing/defocusing properties of the enhanced wake. Almost-homogeneous plasma is assumed to avoid the subtleties associated with the wake excitation in plasma channels [13].

Taking the curl of the Lenz's law, substituting into the resulting formula the time derivative of the Ampere's law, and the using the equation of motion for the linear plasma flow, we obtain

$$
\left(\frac{\partial^{2}}{\partial t^{2}}+\omega_{p 0}^{2}\right) \vec{E}+c^{2} \nabla \times \nabla \times \vec{E}=-4 \pi \frac{\partial \vec{J}_{\mathrm{NL}}}{\partial t}
$$

The density perturbation, related to $\vec{E}$ through the Poisson's equation, can be found by taking the divergence of Eq. (13) and assuming that $\vec{J}_{\mathrm{NL}}=\vec{e}_{z} J_{\mathrm{NL}}\left(\zeta, \vec{x}_{\perp}\right)$ : 


$$
\rho=\frac{J_{\mathrm{NL}}}{c}-\frac{\omega_{p}}{c} \int_{-\infty}^{\zeta} d \zeta^{\prime} \sin \omega_{p}\left(\zeta-\zeta^{\prime}\right) J_{\mathrm{NL}}
$$

Electric field can now be solved component by component. First, the accelerating field is obtained by substituting $\rho$ into Eq. (13):

$$
\left(-\nabla_{\perp}^{2}+k_{p 0}^{2}\right) E_{z}=-4 \pi k_{p 0}^{2} \int_{-\infty}^{\zeta} d \zeta^{\prime} \cos \omega_{p}\left(\zeta-\zeta^{\prime}\right) J_{\mathrm{NL}} .
$$

For a wide beam $\nabla_{\perp}^{2} \ll k_{p 0}^{2}$, and Eq. (15) yields the same expression for $E_{z}$ as Eq. (4). Equation (15) is identical to the one derived by Keinigs and Jones [14] for a beamdriven plasma wakefield accelerator in the limit of $\beta_{g}=1$. The key difference is that in a CBA the current $J_{\mathrm{NL}}$ which drives the enhanced wake is generated through the interaction between the counter-propagating laser pulses and not by an external electron beam. An important advantage of such "virtual beam" is that it can carry positive as well as negative current while propagating in the positive $z$ direction. By changing the sign of the frequency detuning between lasers in a CBA one achieves the same effect as switching from electron to positron driver in a plasma wakefield accelerator.

The transverse component of the electric field $\vec{E}_{\perp}$ can be similarly calculated from Eqs. $(13,14)$. Using Eq. (15) it can be, moreover, shown that $\vec{E}_{\perp}$ is related to $E_{z}$ through

$$
\vec{E}_{\perp}=\frac{c}{\omega_{p 0}^{2}} \vec{\nabla}_{\perp}\left(\frac{\partial E_{z}}{\partial \zeta}\right)
$$

Let's consider the case of $E_{z}>0$ which is peaked on axis, i. e. a positron injector. This corresponds to $\Delta \omega>0$, when the short pulse photons scatter into the pump and deposit positive momentum into plasma electrons. This generates an increase in electron density on axis, pulling positrons towards the axis. Equation (16) leads to the same conclusion: $E_{x, y}<0$. However, $\vec{E}_{\perp}$ is not the only field which affects the transverse particle dynamics. Magnetic field generated by the "virtual beam" defocuses relativistic positrons. The equation for the transverse wakefield $\vec{W}=\vec{E}_{\perp}+\hat{z} \times \vec{B}$ which determines the transverse dynamics of ultra-relativistic particles is derived from the Lenz's law by assuming that both the electric and magnetic fields depend on the $\zeta \approx t-z / c$ :

$$
\vec{W}=-c \int_{-\infty}^{\zeta} \vec{\nabla}_{\perp} E_{z}
$$

Equation (17), which is the Panowski-Wenzel theorem, indicates that whenever $\left|E_{z}\right|$ is peaked on axis, the transverse wake defocuses the particles. Note that this conclusion can only be drawn for snow-plow acceleration which takes place inside the leading portion of the enhanced wake where the sign of $E_{z}$ does not change. If one were to use the wakefield shown in Fig. 1(iii) for accelerating positrons in the second half-period of the wake (counting from the head of the laser pulse), the integral of the gradient of the accelerating field $E_{z}$ in the RHS of Eq. (17) is positive, so that $W<0$ in that region. If, however, one were to use the same wake for snow-plow acceleration of electrons in the leading portion of the wake, the transverse wake $W$ is still negative, and electrons are defocused.

It appears that to ensure focusing during snow-plow acceleration it is essential to generate a somewhat unusual accelerating field profile which has a local minimum of $\left|E_{z}\right|$ on axis. Assuming that the laser spotsize is much larger than $k_{p}^{-1}$, we find that the transverse profile of $E_{z}$ follows the transverse profile of $J_{\mathrm{NL}}$ according to Eq. (4). Therefore, producing a local minimum of $\left|E_{z}\right|$ requires generating $J_{\mathrm{NL}}$ with a local minimum on axis. Such a current profile would be challenging to produce using a finite emittance external beam driver. It appears that it can be produced in a CBA if plasma density has a minimum on axis (plasma channel).

To see this, consider Eq. (3) for $E_{z}$ which indicates that for a fixed $\Delta \omega$ the magnitude of $E_{z}$ at a given $\zeta$ is proportional to $\omega_{p}$. Frequency detuning $\Delta \omega$ is yet another knob which can be tuned in order to maximize current deposition off axis. We conjecture that by the proper choice of the plasma channel width, frequency detuning, and laser intensity the desired profile of $E_{z}$ can be produced to ensure particle focusing during snow-plow acceleration. Plasma channels, which have been produced in the laboratory $[15,16]$, play another beneficial role of guiding the laser pulse over extended distances much larger than the Rayleigh length. Three-dimensional particle simulations will be used in the future to demonstrate the focusing properties of the CBA injector.

Another way of avoiding particle defocusing is to use a hollow plasma channel. As was demonstrated in earlier publications [17], evacuated channels with plasma outside have attractive accelerating properties, including homogeneous accelerating field inside the channel. According to Eq. (17), transverse wake vanishes inside the hollow channel. Closely following the calculations of Refs. ( [17]) for a flat plasma channel of width $b$, we find two major differences in the wake excitation from that in a homogeneous plasma: (i) the wavelength of the plasma wake is $2 \pi c \sqrt{1+k_{p 0} b} / \omega_{p 0}$, and (ii) the amplitude of the wake is reduced by a factor $\sqrt{1+k_{p 0} b}$ from its value given by Eq. (3).

\section{CONCLUSIONS}

In this paper we presented a qualitative description of the colliding beam accelerator (CBA) in one dimension, followed by the fully three-dimensional calculation of the 
plasma wakes generated by two counter-propagating laser beams. We reviewed the earlier ideas of snow-plow acceleration by the leading edge of the laser pulse and demonstrated how the concepts of CBA and snow-plow acceleration can be combined for particle injection and pulse compression. Some of the limitations of the earlier considered snow-plow accelerators are the large threshold intensity of the laser pulse, inability to accelerate both electrons and positrons, and strong sidescatter of the accelerated particles.

The suggested approach based on a CBA concept gets around these drawbacks. Laser intensity needed for snowplowing is reduced due to the strong nonlinear interaction between counter-propagating laser beams. The sign of the accelerating potential can be readily reversed by changing the frequency detuning between the lasers, enabling acceleration of either positive or negative charges. An example of bunch compression/acceleration is presented. We also conjecture that particle defocusing can be avoided by employing a plasma channel. This idea needs further investigation using three-dimensional particle simulations. Utilizing a hollow plasma channel is yet another possibility to avoid particle defocusing.

This work was supported by the DOE Division of High Energy Physics, DOE contract DE-FG030-98DP00210, and the Deutsche Forschungsgemeinschaft.

[1] T. Tajima and J. M. Dawson, Phys. Rev. Lett. 43, 267 (1979).

[2] E. Esarey, P. Sprangle, J. Krall, and A. Ting, IEEE
Trans. Plasma Science 24, 252 (1996), and references therein.

[3] P. Chen, J. M. Dawson, R. W. Huff, and T. Katsouleas, Phys. Rev. Lett. 54, 693 (1985).

[4] G. Shvets, N. J. Fisch, A. Pukhov, and J. Meyer-terVehn, Phys. Rev. E 60, 2218 (1999).

[5] C. M. McKinstrie and E. A. Startsev, Phys. Rev. E 54, 1070 (1996).

[6] C. Du and Z. Xu, "Particle acceleration by a leading edge of a laser pulse in a plasma", submitted to Europh. Letters (1999).

[7] D. Umstadter, J. K. Kim, and E. Dodd, Phys. Rev. Lett. 78, 2073 (1996).

[8] E. Esarey, R. F. Hubbard, W. P. Leemans, A. Ting, and P. Sprangle, Phys. Rev. Lett. 79, 2682 (1997).

[9] A. Pukhov and J. Meyer-ter-Vehn, APS Bulletin 41, 1502 (1996).

[10] J.-M. Rax and N. J. Fisch, Phys. Fluids B 5, 2578 (1993).

[11] J. Dawson, From Particles to Plasmas, Addison-Wesley, Reading, MA (1988); C. J. McKinstrie and M. Yu, Phys. Fluids B 3, 3041 (1991).

[12] P. Sprangle, E. Esarey, and A. Ting, Phys. Rev. A 41, 4463 (1990).

[13] G. Shvets and X. Li, Phys. Plasmas 6, 591 (1999).

[14] R. Keinigs and M. Jones, Phys. Fluids 30, 2

[15] C. G. Durfee III and H. M. Milchberg, Phys. Rev. Lett. 71, 2409 (1993); C. G. Durfee III, J. Lynch, and H. M. Milchberg, Phys. Rev. E 51, 2368 (1995).

[16] Y. Ehrlich, C. Cohen, A. Zigler, J. Krall, P. Sprangle, E. Esarey, Phys. Rev. Lett. 77, 4186 (1996); A. Zigler, Y. Ehrlich, C. Cohen, J. Krall, P. Sprangle, J. Opt. Soc. Amer. B 13, 68 (1996).

[17] T. C. Chiou, T. Katsouleas, C. Decker, W. B. Mori, J. S. Wurtele, G. Shvets, and J. J. Su, Phys. Plasmas 2, 310 (1995); G. Shvets, J. S. Wurtele, T. C. Chiou, and T. Katsouleas, IEEE Trans. Plasma Sci. 24, 351 (1996). 
The Princeton Plasma Physics Laboratory is operated by Princeton University under contract with the U.S. Department of Energy.

\author{
Information Services \\ Princeton Plasma Physics Laboratory \\ P.O. Box 451 \\ Princeton, NJ 08543
}

Phone: 609-243-2750

Fax: 609-243-2751

e-mail: pppl_info@pppl.gov

Internet Address: http://www.pppl.gov 